\title{
Electrophoresis test prevalence, requesting patterns, yield and related bone marrow biopsy findings at a South African tertiary hospital: A 5-year retrospective audit
}

\author{
N Naidoo, ${ }^{1} \mathrm{MB}$ BCh, MPH; R T Erasmus, ${ }^{1}$ MBBS, FMC Path, FACB, DABCC, DHSM; R Grewal, ${ }^{2}$ MB ChB, FC Path (Haem), \\ MMed (Haem Path); A E Zemlin, ${ }^{1}$ MB ChB, FC Path (Chem), MMed (Chem Path) \\ ${ }^{1}$ Division of Chemical Pathology, Department of Pathology, National Health Laboratory Service and Faculty of Medicine and Health Sciences, \\ Stellenbosch University, Cape Town, South Africa \\ ${ }^{2}$ Division of Haematopathology, Department of Pathology, National Health Laboratory Service and Faculty of Medicine and Health Sciences, \\ Stellenbosch University, Cape Town, South Africa
}

Corresponding author: A E Zemlin (azemlin@sun.ac.za)

\begin{abstract}
Background. Studies of electrophoresis testing (serum protein electrophoresis (SPE), urine protein electrophoresis (UPE), immunofixation electrophoresis (IFE)) in a South African (SA) pathology laboratory setting are limited.

Objectives. To evaluate the prevalence, testing pattern and yield of electrophoresis tests performed over a 5-year period in a tertiary academic laboratory and to relate these findings to bone marrow biopsy findings in a few selected cases.

Methods. This was a retrospective audit of all SPE, UPE and IFE tests performed on new and follow-up adult patients (aged $\geq 18$ years) from 2010 to 2015, using data from the Tygerberg Academic Hospital (Cape Town, SA) National Health Laboratory Service hospital information system database. A subgroup analysis of all patients with negative serum (SIFE) and/or urine immunofixation (UIFE) tests who had concurrent bone marrow biopsies close to the time of IFE testing was also performed.

Results. A total of 5086 SPE tests were performed (44.3\% were follow-up tests, and of these patients 13.8\% had SIFE tests); 1299 UPE tests were performed (23.3\% were follow-up tests, and of these patients $33.6 \%$ had UIFE tests). The mean ages of patients who had SIFE and UIFE tests were 59 years (standard deviation (SD) 14.2) and 60 years (SD 15), respectively. The female-to-male ratio was 1.1:1 for both SIFE and UIFE. The negative test yields for SIFE and UIFE were $31.3 \%$ and $52.1 \%$, respectively. Bone marrow biopsy findings for patients with negative SIFE tests identified 8 out of the 20 biopsies (40.0\%) as positive for myeloma.

Conclusion. This audit provides baseline data on the prevalence of test requests, their source and the yield of electrophoresis testing in our laboratory. An increasing trend in SIFE and UIFE was evident.
\end{abstract}

S Afr Med J 2017;107(3):270-273. DOI:10.7196/SAMJ.2017.v107i3.10960

Serum protein electrophoresis (SPE), urine protein electrophoresis (UPE), immunofixation electrophoresis (IFE) and serum-free lightchain (SFLC) analysis are important tests used to diagnose and monitor monoclonal gammopathy in B-cell disorders such as multiple myeloma and monoclonal gammopathy of unknown significance (MGUS). ${ }^{[1,2]}$

An audit of SPE tests in a UK hospital servicing a population of 759000 people found that 10557 SPE tests were conducted in 2011. A sub-study ( $n=145)$ of appropriateness of SPE test requests compared each case history with set audit standards and found that $26 \%$ of requests for SPE tests were inappropriate, with the primary care clinical discipline being the largest source of inappropriate requests. Most of the appropriate SPE test requests were from clinical haematology, renal medicine, rheumatology and geriatric clinical disciplines. $^{[3]}$

A cross-sectional study of inpatient SPE and UPE tests in a tertiary hospital in New South Wales, Australia, servicing a population of over 1 million, found that 29607 SPE and 14374 UPE tests were performed over a 5 -year period. The mean age of the patients tested was 60 years (standard deviation (SD) 19), with $61 \%$ females. Results were positive for paraprotein in $6.7 \%$ of SPE and $0.3 \%$ of UPE tests. ${ }^{[4]}$

A Scottish study investigating the utility of SPE as a screening test specifically for renal outpatients over a 2 -year period $(N=2544$ new patients) reported 1608 tests performed. Approximately 2.5\% of patients had abnormal SPEP results, of whom 2.5\% had MGUS and only one developed multiple myeloma on subsequent follow-up. ${ }^{[5]}$

Studies on the prevalence of electrophoresis testing in a South African (SA) pathology laboratory setting are limited. A recent retrospective audit at Inkosi Albert Luthuli Central Hospital (IALCH), Durban (a quaternary public sector hospital servicing a population of 10 million people in KwaZulu-Natal (KZN) Province), documented 1260 SPE tests and 304 IFE tests performed between February 2011 and July 2011. The positive test yield from IFE testing for the presence of a paraprotein was $46.5 \%$, revealing the need for IFE testing following identification of a suspicious SPE pattern. ${ }^{[6]}$

A retrospective analysis of medical aid claims for the utilisation of a range of pathology procedures from six private sector pathology laboratories across SA between 2003 and 2005 showed an increase in the number of SPE tests performed across increasing age groups in all laboratories investigated. The frequency of SPE testing varied between the laboratories, ranging between $8.7 \%$ and $55 \%$ of all tests requested and indicating inappropriate test utilisation in some cases. ${ }^{[7]}$

Globally, healthcare systems are under pressure to reduce costs while continuing to provide quality services. Laboratory medicine has been targeted as a potential source of savings, with the implementation of principles of demand management and the 
efficient use of laboratory tests used as a means of cost reduction and viewed as a critical function of laboratory managers. ${ }^{[8]}$

\section{Objectives}

To evaluate the prevalence, testing pattern and yield of electrophoresis tests performed over a 5-year period in a tertiary academic laboratory in SA, and relate these findings to bone marrow biopsy findings in a few selected cases.

\section{Methods \\ Study design}

The study was a retrospective audit using data from the Tygerberg Academic Hospital (TAH) National Health Laboratory Service (NHLS) hospital information system database from 1 July 2010 to 30 June 2015. TAH is a 1380 -bed tertiary referral academic hospital affiliated to Stellenbosch University, and services approximately half of the population of the Western Cape Province of SA (total population $\sim 6.2$ million) ${ }^{[9]}$ The NHLS chemical pathology laboratory processes $\sim 100000$ chemistry test requests per month for TAH and other public sector hospitals and clinics in the region.

Inclusion criteria were all SPE, UPE and IFE tests conducted on new and follow-up adult patients (aged $\geq 18$ years) presenting to TAH. SPE, UPE and IFE tests conducted on non-TAH referrals and patients aged $<18$ years were excluded.

A subgroup analysis of all patients with negative serum (SIFE) and/or urine immunofixation (UIFE) tests who had concurrent bone marrow biopsies close to the time of IFE testing was also performed to determine the proportion of cases in which myeloma presented in the bone marrow but SIFE and/or UIFE were negative. The bone marrow reports of these patients were assessed for the percentage of plasma cells present, plasma cell tumour burden and light-chain restriction according to international diagnostic criteria for myeloma. ${ }^{[1]}$ A negative IFE result was defined as absence of an immunoglobulin monoclonal band with absent kappa or lambda light-chain restriction.

\section{Ethical considerations}

To ensure patient confidentiality, all personal identifying information on patients was removed, with only laboratory sample numbers used to label the data. Information pertaining to patient samples was restricted to members of the research team. The study was approved by the Stellenbosch University Health Research Ethics Committee (ref. no. N16/01/002) and was in accordance with the 2013 Declaration of Helsinki.

\section{Laboratory methods}

The SPE, UPE and IFE tests were performed on the Sebia Hydrasys 2 (Sebia, USA) semiautomated electrophoresis system using agarose gels. Our laboratory is accredited by the South African National Accreditation System and subscribes to internal and external proficiency testing schemes, namely Biorad and the Royal College of Pathologists of Australia.

The bone marrow biopsy procedures and reporting were performed in the Department of Haematopathology at TAH in accordance with International Council for Standardisation in Haematology guidelines. ${ }^{[10]}$

\section{Data analysis}

Data were analysed using descriptive statistical techniques using Microsoft Excel version 14 (USA) and SPSS version 20 (USA) statistical software. Follow-up tests on the same patient were removed.
Results were summarised as the prevalence of testing, source of test requests and test yield by test, year, gender and age. Bone marrow biopsy findings (plasma cell percentage, plasma cell tumour burden, light-chain restriction) in patients with negative SPE and/or UPE tests were tabulated.

\section{Results}

\section{Prevalence of testing}

A total of 5086 SPE tests were performed on 2834 individual patients (44.3\% of tests were follow-up tests). Of these 2834 patients, 391 (13.8\%) had SIFE tests. A total of 1299 UPE tests were performed on 996 individual patients (23.3\% of tests were follow-up tests). Of these 996 patients, 334 (33.6\%) had UIFE tests (Table 1).

The number of SIFE tests performed increased steadily from 60 in 2010 to 118 in 2015, and the number of UIFE tests rose from 57 in 2010 to 89 in 2015 (Fig. 1).

The mean age of patients undergoing SIFE tests was 59 years (SD 14.2), while that of patients undergoing UIFE tests was 60 years (SD 15). The female-to-male ratio was 1.1:1 for both SIFE and UIFE.

\section{Sources of test requests}

The main sources of requests for SIFE tests were the haematology oncology outpatient (19.8\%), orthopaedic inpatient (15.8\%), general medical inpatient (16.3\%), general medical outpatient (15.0\%), medical casualty (10.8\%), neurology inpatient (7.9\%), haematology inpatient $(5.3 \%)$ and orthopaedic outpatient (4.1\%) departments. The main sources of requests for UIFE tests were the general medical inpatient (24.1\%), orthopaedic inpatient (18.2\%), haematology oncology outpatient (11.6\%), general medical outpatient (11.0\%), haematology inpatient (7.4\%), medical casualty (7.1\%), neurology inpatient (5.4\%) and orthopaedic outpatient (2.4\%) departments.

\section{Test yield}

For SIFE testing, $66.2 \%$ of test results were positive for the presence of an immunoglobulin monoclonal band with kappa or lambda light-chain restriction (Table 2). The specialties with the highest positive SIFE test yield were haematology (inpatient $75.0 \%$ and

\begin{tabular}{llll} 
Table & 1. Electrophoresis tests, $\mathbf{2 0 1 0}-\mathbf{2 0 1 5}$ & \\
\hline & $\begin{array}{l}\text { Tests excluding } \\
\text { follow-up } \\
\text { tests, } \boldsymbol{n}\end{array}$ & $\begin{array}{l}\text { Follow-up } \\
\text { tests, } \%\end{array}$ \\
Test & Total tests, $\boldsymbol{N}$ & 2834 & 44.3 \\
\hline SPE & 5086 & 996 & 23.3 \\
UPE & 1299 & 391 & 3.0 \\
SIFE & 403 & 334 & 0.9 \\
UIFE & 337 & &
\end{tabular}

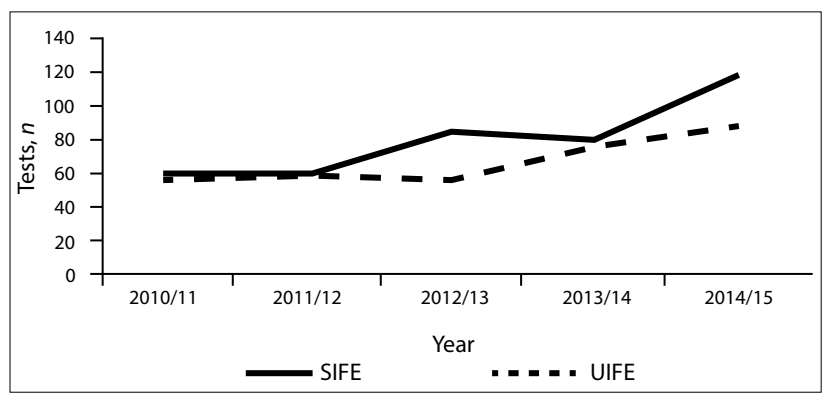

Fig. 1. Numbers of SIFE and UIFE tests, 2010 - 2015. 
Table 2. Yield for all SIFE and UIFE tests, 2010 - 2015

\begin{tabular}{llccl}
\hline Test & Positive results, $\boldsymbol{n}(\boldsymbol{\%})^{*}$ & Negative results, $\boldsymbol{n}(\boldsymbol{\%})^{\dagger}$ & $\begin{array}{l}\text { Oligoclonal/polyclonal } \\
\text { test results, } \boldsymbol{n}(\%)\end{array}$ & $10(2.5)$ \\
\hline SIFE & $267(66.2)$ & $126(31.3)$ & $4(1.2)$ & Total tests, $\boldsymbol{N}$ \\
UIFE & $156(46.7)$ & $174(52.1)$ & 303 \\
*Positive results include the presence of an immunoglobulin monoclonal band with kappa or lambda light-chain restriction or the presence of free kappa and/or lambda light chains. \\
'Negative results include the absence of an immunoglobulin monoclonal band and absence of kappa or lambda light-chain restriction.
\end{tabular}

Table 3. Positive yield for all SIFE and UIFE tests by specialty, 2010 - 2015

\begin{tabular}{|c|c|c|c|c|}
\hline Specialty & $\begin{array}{l}\text { Positive SIFE results, } \\
n(\%)\end{array}$ & $\begin{array}{l}\text { SIFE test positive yield, } \\
\%\end{array}$ & $\begin{array}{l}\text { Positive UIFE results, } \\
n(\%)\end{array}$ & $\begin{array}{l}\text { UIFE test positive yield, } \\
\%\end{array}$ \\
\hline Haematology inpatient & $15(5.6)$ & 75.0 & $10(6.4)$ & 40.4 \\
\hline Haematology outpatient & $67(25.1)$ & 85.8 & $35(22.4)$ & 89.7 \\
\hline Orthopaedics inpatient & $34(13)$ & 54.8 & $18(11.5)$ & 22.2 \\
\hline Orthopaedics outpatient & $13(5.0)$ & 80.6 & $2(1.3)$ & 24.8 \\
\hline General medical inpatient & $38(14.2)$ & 60.3 & $33(21.1)$ & 40.7 \\
\hline General medical outpatient & $28(10.5)$ & 65.1 & $16(10.3)$ & 43.2 \\
\hline Medical casualty & $28(10.5)$ & 68.2 & $12(7.7)$ & 50.2 \\
\hline Neurology inpatient & $13(5.0)$ & 41.9 & $7(4.5)$ & 38.8 \\
\hline Cardiothoracic surgery & $2(0.7)$ & 66.6 & $6(3.9)$ & 85.7 \\
\hline Other specialties & $29(10.9)$ & - & $17(10.9)$ & - \\
\hline Total & $267(100)$ & - & $156(100)$ & - \\
\hline
\end{tabular}

Table 4. Negative SIFE tests and corresponding bone marrow biopsy findings in keeping with the diagnosis of myeloma, $2011-2015$

\begin{tabular}{|c|c|c|c|}
\hline \multirow[b]{2}{*}{ Patient no. } & \multicolumn{3}{|c|}{ Bone marrow findings } \\
\hline & $\begin{array}{l}\text { Plasma cells in bone marrow } \\
\text { aspirate, } \%^{\star}\end{array}$ & $\begin{array}{l}\text { Plasma cells in bone marrow } \\
\text { trephine biopsy }(\mathrm{CD} 138+), \%^{\dagger}\end{array}$ & $\begin{array}{l}\text { Light-chain restriction (kappa and } \\
\text { lambda immunohistochemistry) }\end{array}$ \\
\hline 1 & 7 & $15-20$ & Lambda \\
\hline 2 & 4 & $15-20$ & Kappa \\
\hline 3 & 30 & Clear aggregates ${ }^{\ddagger}$ & Lambda \\
\hline 4 & 16 & 30 & Lambda \\
\hline 5 & Unable to report ${ }^{*}$ & 95 & Not tested \\
\hline 6 & 15 & 80 & Lambda \\
\hline 7 & 4 & $>20$ & Lambda \\
\hline 8 & 14 & $20-30$ & Lambda \\
\hline
\end{tabular}

outpatient $85.8 \%$ ), orthopaedics (outpatient) (80.6\%) and medical casualty (68.2\%) (Table 3$)$.

In contrast, for UIFE testing $46.7 \%$ of results were positive for the presence of an immunoglobulin monoclonal band with kappa or lambda light-chain restriction (Table 2). The haematology outpatient department had the highest positive yield (89.7\%), while the other main specialties requesting UIFE tests had yields $\leq 50 \%$ (Table 3 ).

\section{Negative SIFE and/or UIFE tests and bone marrow biopsy findings}

Of the 403 patients with SIFE tests performed during the 5-year study period, $126(31.3 \%)$ had negative results. Of these patients, 20 (15.9\%) underwent bone marrow biopsies within 6 months of
SIFE testing. Eight of these patients (40.0\%) showed evidence of bone marrow involvement by plasma cells and light-chain restriction on immunohistochemical staining, in keeping with a diagnosis of myeloma $^{[1]}$ (Table 4).

Of the 334 patients with UIFE tests performed during the 5-year study period, 174 (52.1\%) had negative results. Of these patients, 10 (5.7\%) had subsequent bone marrow biopsies, none of which showed findings in keeping with the diagnosis of myeloma. ${ }^{[1]}$

\section{Discussion}

In this study, we performed a 5-year retrospective audit of the prevalence and source of requests for SPE and UPE tests in a tertiary laboratory. We also determined the IFE test yield of these requests 
and compared the negative SIFE and UIFE tests with bone marrow biopsy findings in selected patients from 2010 to 2015 (60 months).

We found that $44.3 \%$ of SPE tests and $23.3 \%$ of UPE tests were followup tests, with an increasing trend in SIFE and UIFE tests performed from 2010 to 2015. This increase could be explained by greater awareness of electrophoresis testing on the part of doctors, an increasing burden of disease, increased requesting for patients with HIV infection, and an increasingly ageing population in the Western Cape. ${ }^{[9]}$

The negative test yields for SIFE and UIFE were $31.3 \%$ and $52.1 \%$, respectively. Of the patients with a negative SIFE test who had a bone marrow biopsy, $40.0 \%$ had bone marrow biopsy features in keeping with the diagnosis of myeloma. No patient with a negative UIFE test who had a bone marrow biopsy had features in keeping with the diagnosis of myeloma.

A previous audit at IALCH found that 1260 SPE tests and 304 SIFE tests were performed over a 6-month period (February 2011 July 2011). ${ }^{[6]}$ Our audit found that 498 SPE and 36 SIFE tests were performed at TAH over the same time period. On average, $509 \mathrm{SPE}$ and 40 SIFE tests were done every 6 months at TAH between 2010 and 2015. The relatively smaller number of tests at TAH could be explained by IALCH's being the only centre performing electrophoresis for KZN, compared with two centres in the Western Cape (TAH and Groote Schuur Hospital). In addition, there is no gatekeeping function for IFE testing at IALCH, so it may be ordered for inappropriate indications. Our study also excluded samples from referral hospitals and clinics from the analysis. The positive test yield for SIFE in our laboratory was higher than at IALCH $(66.2 \% \mathrm{v}$. 46.5\%) ${ }^{[6]}$ Current practice in our laboratory includes a pathologist gatekeeping function for SIFE and UIFE testing based on suspicious SPE and UPE results, respectively. In comparison, the study by Thakkinstian et al. ${ }^{[4]}$ showed positive results for paraprotein in $6.7 \%$ of SPE and $0.3 \%$ of UPE tests, while Doyle et al..$^{[5]}$ had positive SPE findings in $2.5 \%$ of renal outpatients in their hospital.

Our laboratory performed fewer SPE tests in relation to the population served compared with centres in more developed countries. ${ }^{[3-5]}$ For example, $10557 \mathrm{SPE}$ tests were performed in a UK hospital for a population of 759000 in 2011, ${ }^{[3]}$ compared with 1013 tests at TAH for a population of $\sim 3.6$ million in the same year.

Comparing our study with the multi-laboratory study (2003 - 2005) by Pretorius, ${ }^{[7]}$ which showed an increasing trend in test requests with increasing age groups up to $>70$ years, we found a similar trend in our study up to $\sim 63$ years of age for all tests. SPE tests constituted the majority (71.3\%) of all electrophoresis tests performed, suggesting that SPE test requesting patterns have not changed in the past decade, with the exception of fewer tests now being performed in the age group $>70$ years compared with 60 - 69-year-olds.

The patients in our study with negative SIFE and UIPE tests probably had bone marrow biopsies performed owing to strong clinical suspicion or because they had symptoms of myeloma despite negative IFE results. Our finding that only $15.9 \%$ of patients with negative SIFE tests had bone marrow biopsies, of whom $40.0 \%$ (6.3\% of all negative SIFE tests) had positive bone marrow involvement of plasma cells, highlights the value of clinical findings and other test results in the initial diagnosis of myeloma. This pattern is also suggestive of non-secretory myeloma, where the presence of serum or urine monoclonal protein is not mandatory for diagnosis of myeloma and further diagnostic confirmation with SFLC analysis is necessary. ${ }^{[1]}$ Bone marrow biopsy in combination with SIFE and UIFE is also used to monitor response to therapy for myeloma. ${ }^{[11]}$

Other diagnostic modalities for improving the accuracy of diagnosis of plasma cell disorders include SPE testing combined with SFLC testing as first-line investigations, which have shown $100 \%$ sensitivity and $97 \%$ specificity for the detection of plasma cell disorders ${ }^{[12]}$ and the use of a clinical decision rule for SPE and UPE testing using age ( $\geq 60$ years), gender, haemoglobin concentration $(<12.1 \mathrm{~g} / \mathrm{dL})$, globulin level $(>41 \mathrm{~g} / \mathrm{L})$ and estimated glomerular filtration rate $\left(<60 \mathrm{~mL} / \mathrm{min} / 1.73 \mathrm{~m}^{2}\right)$, which has been shown to have an area under the curve of 0.81 after validation. ${ }^{[4]}$

\section{Study limitations and strengths}

This study had several limitations. Unfortunately the time of presentation was not always available, so we were not able to calculate the time taken to reach a diagnosis. A further potential limitation was the subjectivity associated with the interpretation of electrophoresis test results and the fact that the tests were interpreted by numerous pathologists. Test request information and background clinical information were often incomplete, so the appropriateness of testing by clinical discipline could not be assessed. However, strengths were that this audit was performed over a long time period of 5 years and only one method was used for SPE and UPE testing.

\section{Conclusion}

This audit provides baseline data on the prevalence of electrophoresis testing, the source of test requests and test yield in our laboratory, providing useful data for future studies involving plasma cell disorders. The practice of gatekeeping of IFE testing at our hospital has shown benefits, with relatively few SIFE tests performed and higher test result yields compared with another SA hospital of similar size. Against the background of increasing trends in IFE testing at our hospital, the proportion of negative IFE test results, particularly for UIFE, emphasises the value of clinical evaluation when interpreting electrophoresis results.

Acknowledgements. We thank the NHLS Tygerberg Hospital for use of their database and Mr W Kleynhans (IT Department) for data access and retrieval.

Disclaimer. The views expressed in this article are the authors' own and not the official position of the NHLS and Stellenbosch University.

\footnotetext{
1. Rajkumar SV, Dimopoulos MA, Palumbo A, et al. International Myeloma Working Group updated criteria for the diagnosis of multiple myeloma. Lancet Oncol 2014;15(12):e538-e548. http://dx.doi. org/10.1016/s1470-2045(14)70442-5

2. Dispenzieri A, Kyle R, Merlini G, et al. International Myeloma Working Group guidelines for serum-free light chain analysis in multiple myeloma and related disorders. Leukemia 2009;23(2):215-224. http:// dx.doi.org/10.1038/leu.2008.307

McTaggart MP, Kearney EM. Evidence-based use of serum protein electrophoresis in laboratory medicine. Clin Chem Lab Med 2013;51(6):el13-el15. http://dx.doi.org/10.1515/cclm-2012-0711

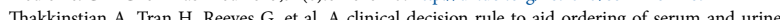
Thakkinstian A, Tran $\mathrm{H}$, Reves $\mathrm{G}$, e al. A clinical decision rule to aid ordering of serum and urine protein electrophoresis for case-finding of paraproteins in hospitalized inpatients. J Gen Intern Med

5. Doyle A, Soutar R, Geddes CC. Multiple myeloma in chronic kidney disease: Utility of discretionary screening using serum electrophoresis. Nephron Clin Pract 2009;111(1):c7-c11. http://dx.doi. org/10.1159/000175798

6. Gounden V, Rampursat Y. An audit of immunofixation requesting practices at a South African referral laboratory: Original research. Afr J Lab Med 2014;3(1):1-5. http://dx.doi.org/10.4102/ajlm.v3i1.91

7. Pretorius C. Utilisation of pathology procedures in the South African private pathology sector between 2003 and 2005. S Afr Med J 2007;97(1):51-57. http://www.samj.org.za/index.php/samj/article/view/419/0 (accessed 9 January 2017)

8. Fryer AA, Smellie WS. Managing demand for laboratory tests: A laboratory toolkit. J Clin Pathol 2013;66(1):62-72. http://dx.doi.org/10.1136/jclinpath-2011-200524

9. Statistics South Africa. Mid-year Population Estimates 2015. Statistical Release P0302. Pretoria: SSA, 2015. https://www.statssa.gov.za/publications/P0302/P03022015.pdf (accessed 9 January 2017). 10. Lee SH, Erber WN, Porwit A, et al. ICSH guidelines for the standardization of bone marrow specimens and reports. Int J Lab Hematol 2008;30(5):349-364. http://dx.doi.org/10.1111/j.1751-553X.2008.01100.x

1. Chee $\mathrm{CE}$, Kumar S, Larson DR, et al. The importance of bone marrow examination in determining complete response to therapy in patients with multiple myeloma. Blood 2009;114(13):2617-2618. http:// dx.doi.org/10.1182/0lood-2009-01-198788

12. McTaggart MP, Lindsay J, Kearney EM. Replacing urine protein electrophoresis with serum free light chain analysis as a first-line test for detecting plasma cell disorders offers increased diagnostic accuracy and potential health benefit to patients. Am J Clin Pathol 2013;140(6):890-897. http://dx.doi.org/10.1309/ ajcp25ihylewcahj
} 